\title{
The Effects of Glucocorticoids on Bone Strength in Children with Renal Diseases
}

\author{
Jun Aoyagi, ${ }^{1}$ Takahiro Kanai, ${ }^{1,}$ Takane Ito, ${ }^{1}$ Jun Odaka, ${ }^{1}$ Takashi Saito, ${ }^{1}$ Hiroyuki Betsui, ${ }^{1}$ and Takanori \\ Yamagata $^{1}$ \\ ${ }^{1}$ Department of Pediatrics, Jichi Medical University, 3311-1 Yakushiji Shimotsuke Tochigi 329-0498, Japan \\ "Corresponding author: Takahiro Kanai, Department of Pediatrics, Jichi Medical University, 3311-1 Yakushiji Shimotsuke Tochigi 329-0498, Japan. Tel: +81-285587366, Fax: \\ +81-285446123, E-mail: tkanai@jichi.ac.jp
}

Received 2016 April 27; Revised 2016 June 29; Accepted 2016 October 23.

\begin{abstract}
Background: We evaluated the effect of glucocorticoids (GCs) on bone strength via bone mineral density (BMD) scores and serum alkaline phosphatase (S-ALP) levels in children with idiopathic steroid-sensitive nephrotic syndrome (ISSNS) or IgA nephropathy (IgAN).

Methods: Sixteen children with ISSNS and 13 with IgAN were eligible for this study. The BMD and S-ALP levels were measured before the initiation of steroid treatment (STx), one month after initiation of STx and one month after terminating STx (Phases 0, 1 and 2). For IgAN, scores and levels were measured before the initiation of STx (Phase 0) and 1, 3, 6, and 12 months after the initiation of STx (Phases 1, 2, 3, and 4).

Results: In ISSNS, the BMD and S-ALP levels were significantly lower in Phase 1 than in Phase 0 ; however, scores and levels returned to baseline in Phase 2. In IgAN, BMD was significantly lower in Phase 2 than in Phase 0 while S-ALP levels were significantly lower in Phases 1, 2, and 3 compared to Phase 0 . No significant difference was observed between Phase 0 and Phase 4 .

Conclusions: In ISSNS, bone strength recovered one month after terminating STx. In IgAN, bone strength recovered 10 months after tapering of STx.
\end{abstract}

Keywords: ALP, BMD, Glucocorticoids, IgA Nephropathy, ISSNS

\section{Background}

Glucocorticoids (GCs) are essential treatments for idiopathic steroid-sensitive nephrotic syndrome (ISSNS) or IgA nephropathy (IgAN), with good clinical results $(1,2)$. On the other hand, they cause bone fragility, and in some cases, fracture (3). The GCs directly inhibits the osteoblastic function by affecting GC receptors on the cells (4) and decreases the lifespan of osteoblasts by apoptosis (5). In addition, GCs decrease the recruitment of osteoblasts and osteoclasts from progenitor cells (6). The bone strength in children is most affected by GCs because during the childhood and adolescence years bone formation occurs faster than bone loss to build strong bones.

It is still unclear that how severely the GCs affect bone strength, especially in children. There is no guideline for preventing fractures caused by glucocorticoid-induced osteoporosis. Therefore, pediatricians may discourage children under steroid treatment (STx) from participating in physical education. One of the determinants of bone strength is bone mineral density (BMD). The serum alkaline phosphatase (S-ALP) level can also provide information on bone strength. In this study, we examined the ef- fects of GCs on bone strength by evaluating BMD scores and S-ALP levels.

\section{Objectives}

The aim of this study was to contribute to providing a guideline about a physical education that could prevent fractures caused by glucocorticoid-induced osteoporosis in children who received GCs.

\section{Methods}

The study protocol was approved by the ethics committee of Jichi Medical University.

The records of children treated at the Jichi pediatric university hospital from September 2007 to September 2012 were analyzed retrospectively. Sixteen children with ISSNS (12 boys and 4 girls, median age 5.5 years, range 1 - 13 years), and 13 with IgAN ( 6 boys and 7 girls, median age 12 years, range 8 - 15 years) were included in this study. The ISSNS patients were all in their first onset. The definition of NS according to the international study of kidney disease in children (ISKDC) criteria is as follows: Proteinuria 
$>40 \mathrm{mg} / \mathrm{m}^{2}$ per hour and hypoalbuminemia $<2.5 \mathrm{~g} / \mathrm{dL}$. The diagnosis of ISSNS was made when remission of NS was achieved within four weeks of initiation of STx. The IgAN patients had not received any prior GC treatments. IgAN was defined by the presence of diffuse dominant or codominant mesangial deposits of immunoglobulin $\mathrm{A}(\operatorname{IgA})$, with mesangial cellular proliferation in renal tissue.

The ISSNS patients were treated with prednisolone (2 $\mathrm{mg} / \mathrm{kg}$ per day, maximum $60 \mathrm{mg}$ per day) in three divided doses for four weeks, followed by $1.3 \mathrm{mg} / \mathrm{kg}$ in one single-morning dose on alternate days for another four weeks. Then prednisolone was terminated eight weeks after STx initiation. Steroid-dependent and steroid-resistant patients were excluded from the study. None of the patients received immunosuppressive agents, or any drugs that influenced bone strength such as Ca, vitamin D, and alendronate.

The IgAN patients underwent tonsillectomy followed by three courses of steroid pulse therapy. Each course consisted of methylprednisolone $(20 \mathrm{mg} / \mathrm{kg}$, maximum 500 $\mathrm{mg}$ ) per dose on three consecutive days per week, followed by prednisolone ( $1 \mathrm{mg} / \mathrm{kg}$ per day, max $30 \mathrm{mg}$ per day). They were then treated with prednisolone $(1 \mathrm{mg} / \mathrm{kg})$ every other day for 4 - 9 months (median 5 months) until they reached a remission phase and were negative for proteinuria and hematuria.

We evaluated bone strength by BMD scores and S-ALP levels. In the ISSNS group, BMD scores and S-ALP levels were examined in Phase 0 before the initiation of STx; in Phase 1 one month after the initiation of STx; and in Phase 2 one month later after terminating STx, that is, two months after the initiation of STx. The BMD of the lumbar spine (L2 L4) was measured using dual-energy $x$-ray absorptiometry scans (The Discovery A, Hologic, USA). In the IgAN group, the BMD scores and S-ALP levels were examined as follows: Phase 0 , before the initiation of STx, in Phases 1, 2, and 3 at one, three and six months, respectively, after the initiation of STx and in Phases 4,12 months after the initiation of STx (10 months after tapering of STx). The patients had no restrictions on physical education. The variation of bone strength was evaluated by comparing pre-treatment BMD scores and S-ALP levels with those at each month.

Statistical analyses were performed using JMP Software 8 (SAS Institute Inc. USA). Paired t-test was used for comparisons. Results are presented as the mean $\pm \mathrm{SE}$, and a Pvalue of less than 0.05 was considered statistically significant.

\section{Results}

4.1. Comparison of Bone Strength in Phases 0 - 2 in ISSNS Patients

In ISSNS patients, the BMD scores and S-ALP levels were significantly lower in Phase 1 (BMD score, $-0.062 \pm 0.013, \mathrm{P}$ $=0.0004$; S-ALP level, $-0.465 \pm 0.053, \mathrm{P}<0.0001$ ) than in Phase 0 . The BMD scores and S-ALP levels were not significantly different between Phase 0 and Phase 2 (BMD score, $-0.0022 \pm 0.010, \mathrm{P}=0.83$; S-ALP level, $0.158 \pm 0.092, \mathrm{P}=0.11$, respectively) (Figure $1 \mathrm{~A}$ and $\mathrm{B}$ ).

\subsection{Comparison of Bone Strength in Phases 0 - 4 in IgAN Patients}

In IgAN participants, only the BMD score in Phase $2(-$ $0.025 \pm 0.0096$ ) was significantly lower than in Phase $0(P$ $=0.025)$. The S-ALP levels were significantly lower in Phases 1,2 , and $3(-0.431 \pm 0.030,-0.264 \pm 0.053,-0.221 \pm 0.079$, respectively) than in Phase $0(\mathrm{P}<0.0001, \mathrm{P}=0.0006, \mathrm{P}$ $=0.019$, respectively). No significant differences were detected between Phase 0 and Phase 4 in BMD scores and $S$ ALP levels $(0.008 \pm 0.019, \mathrm{P}=0.68,-0.077 \pm 0.072, \mathrm{P}=0.31)$ (Figure 2A and B).

4.3. Comparison of BMD Scores and S-ALP Levels in Phases 0 - 1 between ISSNS and IgAN Patients

The variation rate of BMD scores in Phase 1 in ISSNS was significantly lower than that of IgAN $(P=0.0065)$. No significant difference was observed between ISSNS and IgAN in the variation rate of s-ALP levels in Phase $1(\mathrm{P}=0.58)$.

\subsection{The Total Dose of Steroid}

The total dose of steroid administration was higher in IgAN participants $(8.7 \mathrm{mg} / \mathrm{kg}$ per day in prednisolone equivalents for one month; max $6195 \mathrm{mg}$ ) than in ISSNS participants ( $2 \mathrm{mg} / \mathrm{kg}$ per day for one month; max 1680 $\mathrm{mg})$.

\section{Discussion}

In this study, we showed the effect of GCs on bone strength based on BMD scores and S-ALP levels in children with ISSNS or IgAN.

Sbrocchi et al. (7) reported vertebral fractures despite normal spine BMD in a boy with NS. This report suggested that observation of BMD alone might not be sufficient to evaluate bone strength of children with NS in GC treatment. Biyikli NK et al. (8) reported that high-dose corticosteroids treatment causes a decrease in bone formation, as shown by the changes in ALP levels. Therefore we examined BMD and serum ALP level at the same time. In this study, BMD scores were also associated with S-ALP levels in ISSNS. 


\section{A}

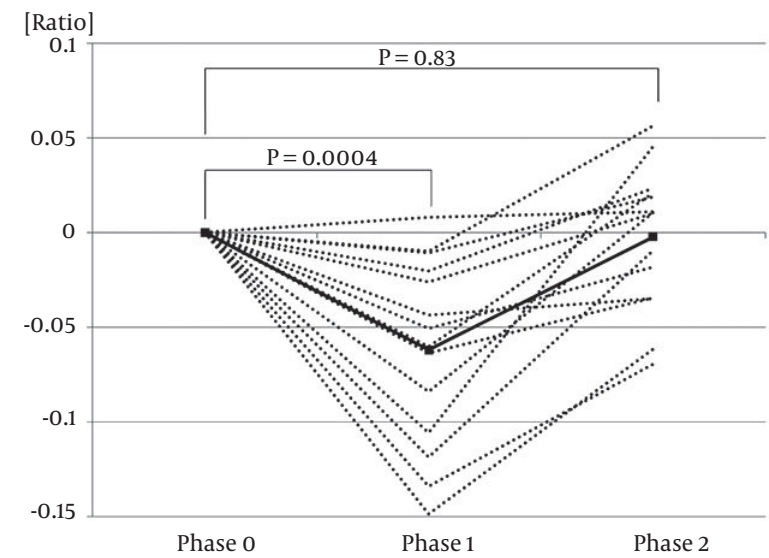

\section{B}

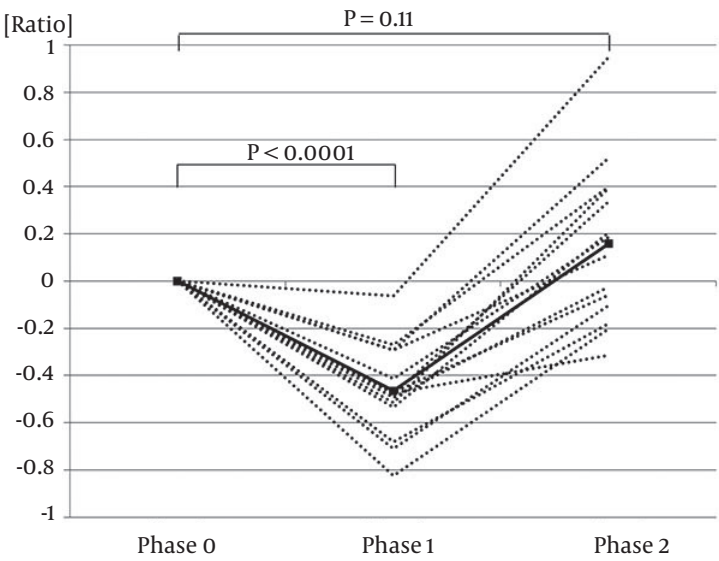

Figure 1. A, The Observation of the Variation Rate in Bone Mineral Density in Phase 0 - 2 in Idiopathic Steroid-Sensitive Nephrotic Syndrome; B, The Observation of the Variation Rate in Serum Alkaline Phosphatase Levels in Phase 0 - 2 in ISSNS

A

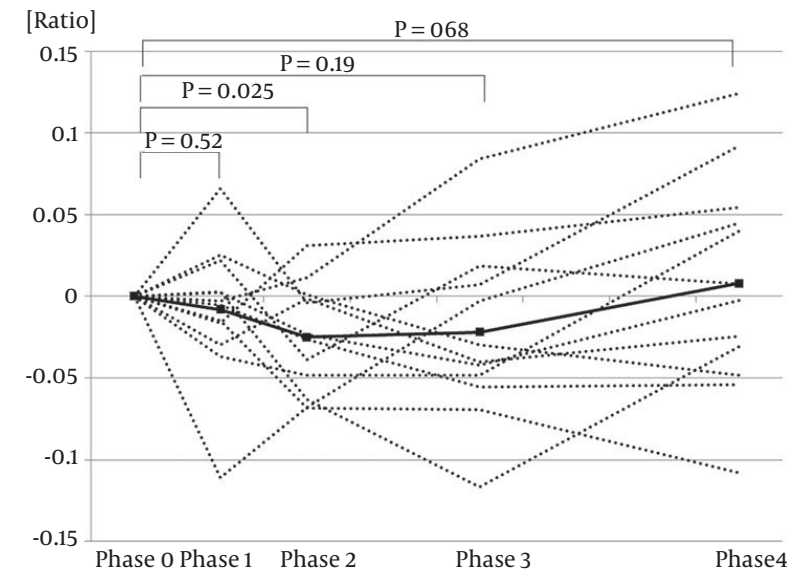

B

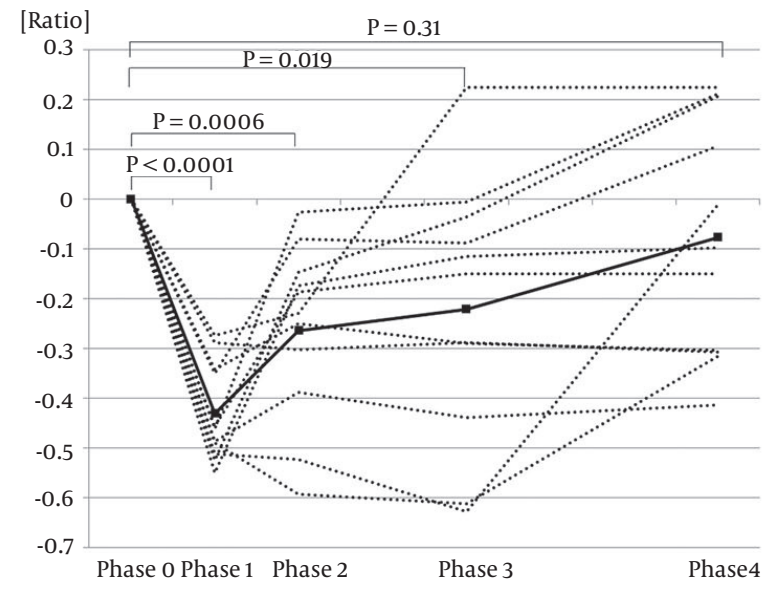

Figure 2. A, The Observation of the Variation Rate in Bone Mineral Density in Phase 0 - 4 in IgA Nephropathy; B, The Observation of the Variation Rate in Serum Alkaline Phosphatase Levels in Phase 0 - 4 in IgAN

In the ISSNS group, the BMD scores and S-ALP levels indicated that bone strength recovered quickly one month after terminating STx. In the IgAN group, the BMD scores and S-ALP levels indicated that bone strength recovered 10 months after tapering of STx. Most of the patients in the IgAN group were adolescents. Bone formation is very active in the adolescent period, so we supposed that the duration for bone strength normalization would be shorter in the IgAN group than in the ISSNS group. However, the IgAN group required a longer normalization period than the ISSNS group. Thus, even low dose GCs in IgAN group from phase 2 to phase 4 affect bone strength in childhood.
There was a difference in the effects of GCs on bone strength at one month (Phase 1) between the ISSNS and IgAN patients. The BMD scores and S-ALP levels were negatively associated in ISSNS patients while the BMD score was not affected in IgAN patients. Children with IgAN were older (median age 12 vs. 5.5 years), and the proportion of girls was higher ( 0.54 vs. 0.25 ) than in the ISSNS patient group although there were no associations between age, sex and BMD scores in ISSNS and IgAN, respectively. During puberty, there is a large increase in lumbar spine BMD score. The influence of puberty on BMD is higher in girls than in boys (9). Therefore, the older girls in puberty may 
have caused the difference in changes of BMD scores between the ISSNS and IgAN patients. Our results suggested that the increase in BMD scores exceeded the negative effect of GCs on BMD scores during puberty.

It is also hypothesized that the effect of pulse GC treatment on bone strength was mild. In a comparative study of the effect of a daily steroid regimen versus a weekly oral pulse steroid regimen on BMD scores in patients with skin disease, the effect of a weekly oral pulse steroid was much smaller than with the daily steroid (10). Another report showed that ALP levels remained unchanged after pulse GC treatment (11).

\subsection{Conclusions}

Bone strength recovered quickly one month after terminating STx in the ISSNS group while even a low dose negatively affected the IgAN group. It was also found that the increase in BMD scores exceeded the negative effect of GCs on BMD scores during puberty. Our report provides an interesting new perspective for the evaluation of bone dysbolism in pediatric renal patients.

\section{References}

1. Hodson EM, Knight JF, Willis NS, Craig JC. Corticosteroid therapy in nephrotic syndrome: a meta-analysis of randomised controlled trials. Arch Dis Child. 2000;83(1):45-51. doi: 10.1136/adc.83.1.45. [PubMed: 10868999].

2. Pozzi C, Andrulli S, Del Vecchio L, Melis P, Fogazzi GB, Altieri P, et al. Corticosteroid effectiveness in IgA nephropathy: long-term results of a randomized, controlled trial.JAm Soc Nephrol. 2004;15(1):157-63. doi: 10.1097/01.ASN.0000103869.08096.4F. [PubMed: 14694168].
3. Phan V, Blydt-Hansen T, Feber J, Alos N, Arora S, Atkinson S. Skeletal findings in the first 12 months following initiation of glucocorticoid therapy for pediatric nephrotic syndrome. Osteoporosis Int. 2014;25(2):627-37.

4. Morrison NA, Shine J, Fragonas JC, Verkest V, McMenemy ML, Eisman JA. 1,25-dihydroxyvitamin D-responsive element and glucocorticoid repression in the osteocalcin gene. Science. 1989;246(4934):1158-61. [PubMed: 2588000].

5. Saag KG. Glucocorticoid-induced osteoporosis. Endocrinol Metabol Clin North Am. 2003;32(1):135-57.

6. Weinstein RS, Jilka RL, Parfitt AM, Manolagas SC. Inhibition of osteoblastogenesis and promotion of apoptosis of osteoblasts and osteocytes by glucocorticoids. Potential mechanisms of their deleterious effects on bone. J Clin Invest. 1998;102(2):274-82. doi: 10.1172/JCI2799. [PubMed: 9664068].

7. Sbrocchi AM, Rauch F, Matzinger M, Feber J, Ward LM. Vertebral fractures despite normal spine bone mineral density in a boy with nephrotic syndrome. Pediatr Nephrol. 2011;26(1):139-42. doi: 10.1007/s00467-010-1652-5. [PubMed: 20922433].

8. Biyikli NK, Emre S, Sirin A, Bilge I. Biochemical bone markers in nephrotic children. Pediatr Nephrol. 2004;19(8):869-73. doi: 10.1007/s00467-004-1537-6. [PubMed: 15206021].

9. Boot AM, de Ridder MA, Pols HA, Krenning EP, de Muinck KeizerSchrama SM. Bone mineral density in children and adolescents: relation to puberty, calcium intake, and physical activity. J Clin Endocrinol Metab. 1997;82(1):57-62. doi: 10.1210/jcem.82.1.3665. [PubMed: 8989233].

10. El-Darouti MA, Mashaly HM, El-Nabarawy E, El-Tawdy AM, Fawzy MM, Salem DS, et al. Comparative study of the effect of a daily steroid regimen versus a weekly oral pulse steroid regimen on morphological changes, blood sugar, bone mineral density and suprarenal gland activity. J Dermatolog Treat. 2012;23(1):4-10. doi: 10.3109/09546634.2010.487887. [PubMed: 20819024].

11. Lems WF, Gerrits MI, Jacobs JW, van Vugt RM, van Rijn HJ, Bijlsma JW. Changes in (markers of) bone metabolism during high dose corticosteroid pulse treatment in patients with rheumatoid arthritis. Ann Rheum Dis. 1996;55(5):288-93. doi: 10.1136/ard.55.5.288. [PubMed: 8660101]. 